\title{
Tools and Methods to Include Health in Climate Change Adaptation and Mitigation Strategies and Policies: A Scoping Review
}

\author{
Ianis Delpla ${ }^{1, *}$, Thierno Amadou Diallo ${ }^{2}$, Michael Keeling ${ }^{2}$ and Olivier Bellefleur ${ }^{2}$ \\ 1 École Supérieure D’aménagement du Territoire et de Développement Régional (ESAD), Université Laval, \\ Pavillon F-A. Savard, 2325, rue des Bibliothèques, local 1612, Québec, QC G1V 0A6, Canada \\ 2 National Collaborating Centre for Healthy Public Policy, Montréal, QC H2P 1E2, Canada; \\ thiernoamadou.diallo@inspq.qc.ca (T.A.D.); michael.keeling@inspq.qc.ca (M.K.); \\ olivier.bellefleur@inspq.qc.ca (O.B.) \\ * Correspondence: Ianis.Delpla@crad.ulaval.ca
}

Citation: Delpla, I.; Diallo, T.A.; Keeling, M.; Bellefleur, O. Tools and Methods to Include Health in Climate Change Adaptation and Mitigation Strategies and Policies: A Scoping Review. Int. J. Environ. Res. Public Health 2021, 18, 2547. https:// doi.org/10.3390/ijerph18052547

Academic Editor: Paul Tchounwou

Received: 24 January 2021

Accepted: 26 February 2021

Published: 4 March 2021

Publisher's Note: MDPI stays neutral with regard to jurisdictional claims in published maps and institutional affiliations.

Copyright: (c) 2021 by the authors. Licensee MDPI, Basel, Switzerland. This article is an open access article distributed under the terms and conditions of the Creative Commons Attribution (CC BY) license (https:/ / creativecommons.org/licenses/by/ $4.0 /)$.

\begin{abstract}
Climate change represents a serious threat to the health and well-being of populations. Today, many countries, regions, and cities around the world are implementing policies and strategies to adapt to climate change and mitigate its effects. A scoping review was performed to identify tools and methods that help integrate health into climate change adaptation and mitigation policies and strategies. The literature search includes scientific and grey literature. The scientific literature was conducted using PubMed, Elsevier Embase, and Web of Science databases. A grey literature web search was performed to complement the results. A total of 35 studies ( 28 from the scientific literature and 7 from the grey literature) were finally included. A large majority of research articles (24/28) and almost all reports (6/7) from the grey literature were published after 2010. Results show that the tools that were found most frequently are the nested models (12/35), health impact assessment (6/35), vulnerability and adaptation assessment (3/35), conceptual frameworks (3/35), and mixed methods (3/35). This review shows an increasing interest in the topic of developing tools to better manage health issues in adaptation and mitigation strategies, with a recent increase in the number of publications. Additional analyses of tools' effectiveness should be conducted in further studies.
\end{abstract}

Keywords: climate change policies; health; tools; adaptation; mitigation

\section{Introduction}

Climate change poses serious threats to human health and well-being, and it contributes to increasing health inequalities between and within countries. The health impacts of climate change can be direct (for example, heat waves, extreme weather, and events such as storms, forest fires, floods, or drought) or indirect through the effects of climate change on ecosystems (for example, agricultural losses, changes in disease distribution patterns, infrastructure disruptions) and on the economy and social structures (for example, migration and conflict) [1-3].

The health effects of climate change can be summarized as follows [4]: heat-related disorders, respiratory disorders, infectious diseases including vector- and water-borne diseases, disruptions to food production and mental health disorders. In 2014, World health Organization (WHO) established that more than 7 million deaths are attributable to air pollution each year [5]. WHO says climate change is likely to lengthen the transmission period of some major vector-borne diseases and change their geographic distribution. Concerning the effects on water-borne diseases, extreme weather events are associated with an increased risk of surface water contamination by protozoa, such as Giardia cysts and cryptosporidium oocysts [6]. Finally, the effects of climate change on mental health is an emerging research topic. These effects will not be felt in the same way by all, but will disproportionately affect vulnerable groups. 
The 2015 Paris Agreement on Climate is seen as an important step towards the global strategy to reduce the risks of climate change [7]. This agreement called upon countries to take ambitious adaptation and mitigation actions and ensure their implementation [8]. Following this, the Intergovernmental Panel on Climate Change appealed for actions to limit the temperature increase to $1.5^{\circ} \mathrm{C}$ to avoid, among others, significant risks to human health [9].

In this context, cities, and countries are developing policies or strategies to reduce greenhouse gas (GHG) emissions (mitigation) and to reduce vulnerability to the effects of climate change (adaptation). In these policies or strategies, it is necessary that health impacts be specifically taken into account in order to achieve both environmental protection and health promotion. However, health impacts are rarely considered by decision-makers involved in developing climate change adaptation and mitigation policies [10]. Actually, some existing tools such as health impact assessment (HIA) can be deployed to help take health issues into consideration when developing climate change-related policies [10].

However, to date and to our knowledge, there has not been a study that systematically reviews the tools and methods that were designed to promote or integrate health into climate change adaptation and mitigation policies or strategies. Selecting such tools and methods will help in the development of specific policies and strategies of adaptation and mitigation that contribute to environmental protection and health promotion.

The purposes of this paper are (1) to conduct a scoping review on climate change studies that focus on the development of tools and methods that help integrate health into climate change adaptation and mitigation policies and strategies, and (2) to assess and select the tools and methods that could be useful for stakeholders and contribute to protecting and promoting health.

\section{Materials and Methods}

\subsection{Search Criteria}

The literature search focused on the identification of all primary studies that aim to develop methods and tools that integrate health into climate change adaptation and mitigation policies and strategies published in English and French in scientific journals and grey literature between January 1990 and September 2019. The strategy used to conduct this review, in accordance with the PRISMA guidelines [11], consisted of grouping keywords that represented (i) the tools (frameworks, methods, etc.), (ii) the climatic events (namely, climate change and its effects: extreme events, etc.), and (iii) the health-related aspects (policies or diseases).

The following keywords and combinations were used for the literature search on Pubmed: (Tool*[TIAB] OR Method[TIAB] OR framework[TIAB] OR "Health Impact Assessment"[MeSH]) AND ("Climate change" [MeSH] OR "Climate change"[TIAB] OR "Climatic change"[TIAB] OR "Global warming"[TIAB] OR “Greenhouse effect"[MeSH:NoExp] OR “Extreme events"[TIAB]) AND ("Public health"[MeSH:NoExp] OR "Public policy"[MeSH:NoExp] OR "Health planning"[MeSH:NoExp] OR “Health policy"[MeSH:NoExp] OR “Air pollution" OR "Aeroallergens" OR "Heat waves" OR "Urban heat island effect" OR "Vector-borne diseases" OR "Waterborne diseases" OR "Water \& food supply" OR "Mental health" OR "Environmental refugees").

The literature search included scientific and grey literature. Keywords, titles, and abstracts were searched in PubMed, Elsevier Embase and Web of Science for scientific literature. For the grey literature search, Québec University databases, websites of the World Health Organization (WHO), the Intergovernmental Panel on Climate Change (IPCC), United Nations (United Nations Environment Programme (UNEP), United Nations Framework Convention on Climate Change (UNFCC)), World Meteorological Organization, ministries of health, and ministries of the environment of the United States, Canada (including state and provincial websites) and France were included. Considering the considerable number of documents published in the grey literature, the geographical area was restricted to North America to focus on regional studies and France to include some relevant studies published in French. There was no restriction on geographical 
location for the scientific literature search. Librarians of Laval University and of the documentation service of the Institut National de Santé Publique du Québec (INSPQQuébec's Public Health Institute) helped the two first authors to design and validate the literature search strategy.

\subsection{Selection Criteria}

In the first selection stage (exclusion criteria), the abstract of each article was read and screened. The papers meeting the following criteria were excluded from our review:

- Studies only presenting the climate change impacts on human health or the health system in qualitative or quantitative terms.

- Studies presenting only health impact assessment tools for extreme events (e.g., droughts, heatwaves, hurricanes).

- Studies presenting tools/methods that assess the health benefits of policies/strategies outside of the scope of climate change adaptation and mitigation.

- Commentaries, editorials, press releases, speeches, review articles, systematic reviews, or meta-analyses.

- Studies not published in English or French.

In a second selection stage (inclusion criteria), papers that remained from the previous stage were fully screened and then retained according to the following criteria:

- Studies that clearly present the link between the health issue and a meteorological factor that could be modified by climate change.

- Studies that aim to assess the health effects of a policy/strategy designed to adapt to or mitigate climate change consequences or reduce greenhouse gas emissions.

- Studies in which tools/methods are presented and described.

Finally, the references sections of the studies identified were screened, and relevant references that were not initially identified were added.

\subsection{Data Extraction}

Selected articles were reviewed and documented for the following information: first author, location, date of publication, type of climatic event, exposure measurement, health outcomes assessed, type and description of mitigation/adaptation strategy, tool name, origin, objective, and description; and strategy's health effects measurements.

\section{Results}

\subsection{Description of Studies Selected}

\subsubsection{Scientific Literature}

The literature search was conducted between 4-7 February 2019; 1718 articles were identified.

The first selection was based on titles, keywords, and abstracts. It should be noted that a majority of studies used models to assess the health impacts of different climate change scenarios without presenting any adaptation or mitigation strategies or tools and methods. These studies were not retained for in-depth review.

After this first selection, 108 articles were retained. Among these articles, 4 were unavailable, 2 were not in French or English, and 1 was a conference proceeding; these were consequently removed. Then, a final selection of 101 articles was retained for in-depth review.

\subsubsection{Grey Literature}

The grey literature review was conducted between 15 February and 15 March 2019. The first selection was made based on titles, keywords, and abstracts. 43 reports were selected for in-depth review. All studies were in English. Table 1 synthesizes the scientific and grey literature search results.

The detailed results of the article and report selection are presented in Figure 1. 
Table 1. Scientific and grey literature review results, number of articles by language and database.

\begin{tabular}{|c|c|c|c|}
\hline Type of Literature- & Source & Number of Documents & Total \\
\hline \multirow{3}{*}{ Scientific literature } & Web of science & 575 & \multirow{3}{*}{1718 * } \\
\hline & Pubmed & 525 & \\
\hline & Embase & 982 & \\
\hline \multirow{6}{*}{ Grey literature } & $\mathrm{WHO}$ & 19 & \multirow{6}{*}{43} \\
\hline & IPCC & 3 & \\
\hline & WMO & 5 & \\
\hline & Ministry of Environment & British Columbia (1); United States (3); California (1) & \\
\hline & Ministry of Health & Ontario (2); British Columbia (1); & \\
\hline & Others & Canada (1); California (1); France (1) & \\
\hline
\end{tabular}

* After duplicate suppression.

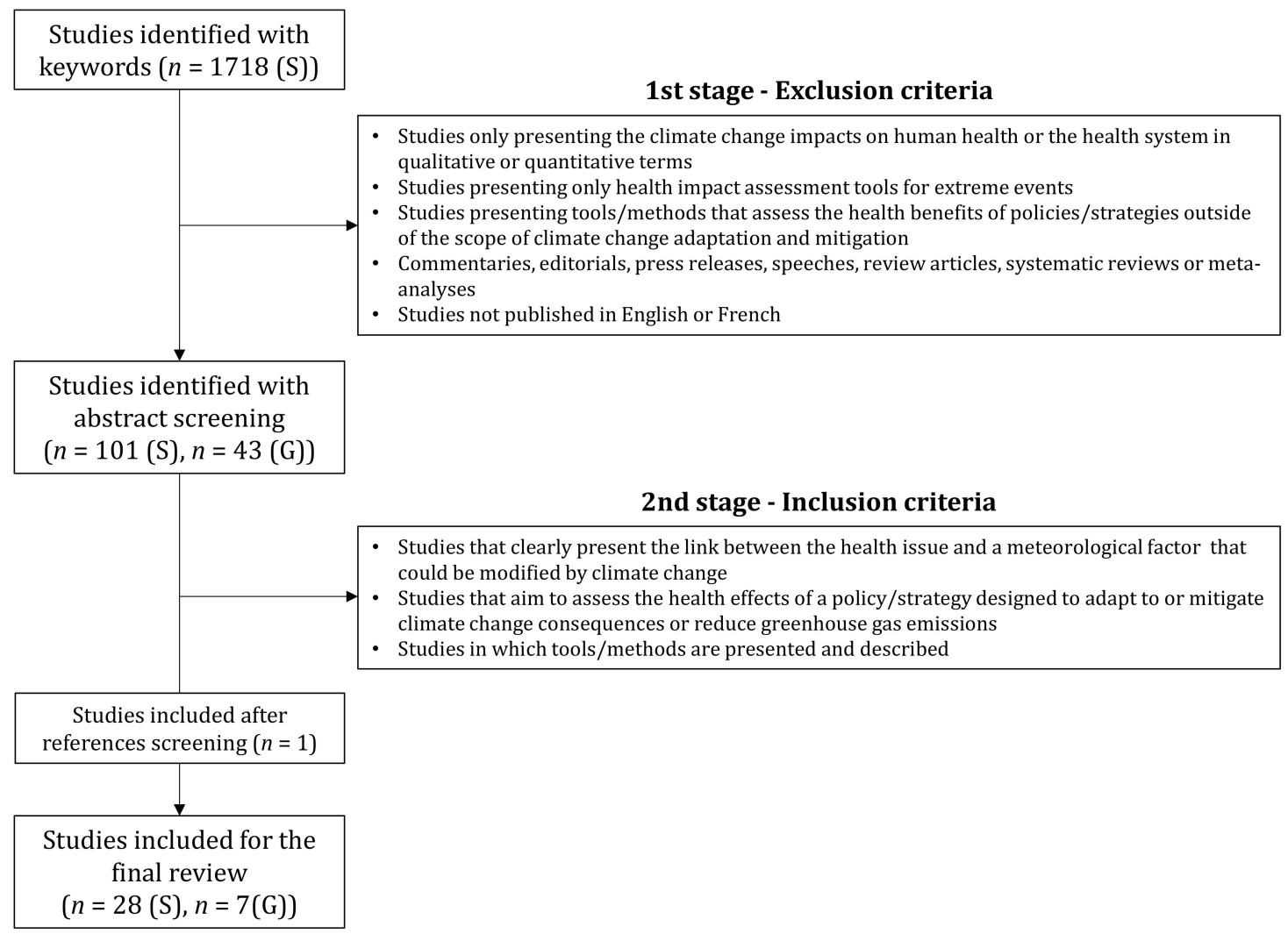

Figure 1. Data research results (S: Scientific literature; G: Grey literature).

\subsection{Description of Tools Selected}

In the scientific literature, the studies identified were conducted mainly in Europe (11), followed by North America (7) and Asia (6), the remaining ones being conducted in Australia (1) and New Zealand (1). There was a majority of mitigation studies that focus on exposure to air pollution (22 of 28), such as particulate matter (PM2.5 and/or PM10). Some studies focus on exposure to air temperature $(n=3)$ or urban heat island $(n=2)$. The main health indicators used are mortality, disability-adjusted life years (DALY), and years of life lost (YLL). Publication dates were between 2008 and 2018, with a large majority of studies (24 of 28) published after 2010. Reports found through the grey literature research have different characteristics than the scientific literature. The WHO is the main organization providing tools aiming at including health in adaptation plans and policies (6 of 7 reports). The reports were published between 2003 and 2018, with almost all after 2010 (6 of 7). 
The different tools that were identified through this literature review were grouped into four main categories: impact assessment tools, adaptation tools, nested models, and conceptual frameworks. We also added one additional category that regrouped mixed methodological approaches. The tools are presented in Table 2.

\subsubsection{Impact Assessment Tools}

Health Impact Assessment (HIA)

HIA has been defined as "a combination of procedures, methods and tools by which a policy, programme, or project may be judged as to its potential effects on the health of a population, and the distribution of those effects within the population" [47]. It consists of five steps: (1) Screening: to decide whether or not a proposal needs an HIA; (2) Scoping: to plan how the HIA should be done; (3) Appraisal: to determine the extent to which the proposal will affect health, the nature of these effects and which population groups will be affected by these effects; (4) Recommendations and reporting: to formulate recommendations for minimizing the negative effects and maximizing the positive effects of a proposal and to present the results of the HIA in a report; (5) Evaluation and monitoring: to review the process and its influence and to monitor what happens once the proposal is put in place [47]. HIA can be used to analyze the potential health impacts of planned climate change policies. For example, this tool can be used to assess the impact on health of climate change mitigation policies at the local level $[19,20]$, to evaluate the climate change resilience that is incorporated into specific building projects [24], or to study the health co-benefits of local climate change mitigation policies in the transport sector [29,34].

\section{Comparative Risk Assessment (CRA)}

The World Health Organization (WHO) defined Comparative Risk Assessment (CRA) as "the systematic evaluation of the changes in population health which result from modifying the population distribution of exposure to a risk factor or a group of risk factors" [48]. This method consists of four steps (adapted from [49]): (1) Identifying health risks associated with exposure; (2) Quantifying the dose-response relationship for a baseline; (3) Defining future exposure scenarios, and (4) Estimating the burden of disease that is attributable to a risk factor and the burden that is avoidable by plausible reductions in the risk factor. CRA has been used to inform climate change mitigation decisions [49]. For example, it has been used to evaluate the potential health co-benefits from the establishment of a mass rapid transit project in Kuala Lumpur [25]; to estimate the health effects of alternative urban land transport scenarios in the context of greenhouse gas emissions reduction in London and Delhi [38]; and to evaluate the health benefits resulting from GHG reduction measures in the electricity generation sector in the European Union (EU), China, and India [28].

\section{Integrated Environmental Health Impact Assessment (IEHIA)}

Integrated environmental health impact assessment (IEHIA) is an inclusive approach for assessing health-related issues associated with environmental changes in order to consider the complexities, interdependencies, and uncertainties of the real world [14]. As [14] indicated in his study, IEHIA derives from risk assessment, environmental impact assessment, HIA, and CRA. The IEHIA process involves four steps [14]: (1) Issue framing: specifying the policy question and developing a conceptual model of the issue to be addressed; (2) Design: defining a detailed protocol for assessment from the conceptual model elaborated in the previous step. This will include defining variables, causal relationships, policy scenarios, models, data, and tools. (3) Execution: modeling and analyzing different scenarios in terms of exposure and health effects and comparing results of the assessment; (4) Appraisal: this step involves synthesizing and interpreting the results; evaluating the outcome actions for the scenarios; and prioritizing the different policy options based on acceptability or effectiveness. 
Table 2. (a) Synthesis of tools from the selected articles: scientific literature. (b) Synthesis of tools from the selected articles: grey literature.

\begin{tabular}{|c|c|c|c|c|c|c|c|c|c|c|c|c|}
\hline \multicolumn{13}{|c|}{ (a) } \\
\hline Author & Year & $\begin{array}{c}\text { Climatic } \\
\text { Event }\end{array}$ & Exposure & Health Issue & $\begin{array}{c}\text { Strategy } \\
\text { (Adapta- } \\
\text { tion/Mitigation) } \\
\end{array}$ & Tool Type & Name & Provider & Tool's Objective & $\begin{array}{l}\text { Scale of } \\
\text { Applica- } \\
\text { tion }\end{array}$ & Area & $\begin{array}{l}\text { Measure of } \\
\text { the Health } \\
\text { Effect }\end{array}$ \\
\hline Abel et al. [12] & 2018 & $\begin{array}{c}\text { Rising } \\
\text { temperatures }\end{array}$ & $\begin{array}{l}\text { Air pollution (PM2.5 } \\
\text { and ozone) }\end{array}$ & $\begin{array}{l}\text { Incidence of premature } \\
\text { mortality and morbidity }\end{array}$ & Mitigation & $\begin{array}{l}\text { Nested } \\
\text { models }\end{array}$ & $\begin{array}{c}\text { EPA's BenMAP } \\
\text { Community Edition } \\
\text { version } 1.3\end{array}$ & US EPA & $\begin{array}{l}\text { BenMAP calculates adverse health outcomes of air } \\
\text { quality changes linked with adaptation in building } \\
\text { energy use }\end{array}$ & Region & $\begin{array}{l}\text { East and } \\
\text { Midwest USA }\end{array}$ & Yes \\
\hline Beaudoin et Gosselin [13] & 2016 & $\begin{array}{c}\text { Rising } \\
\text { temperatures }\end{array}$ & Urban heat islands & Well-being & Adaptation & $\begin{array}{c}\text { Other method- } \\
\text { ological } \\
\text { approach }\end{array}$ & UNSP & $\begin{array}{l}\text { National Institute of } \\
\text { Public Health of } \\
\text { Québec }\end{array}$ & $\begin{array}{l}\text { Assess the effects of Urban Heat Island on well-being } \\
\text { and quality of lifif of residents and ussers. 4criteria } \\
\text { asssessed: beeauty, comfort, coolness, and security }\end{array}$ & City & $\begin{array}{l}\text { Montréal } \\
\text { (Canada) }\end{array}$ & No \\
\hline Briggs [14] & 2008 & UNSP & UNSP & UNSP & UNSP & $\begin{array}{l}\text { Impact } \\
\text { assessment } \\
\text { tool }\end{array}$ & $\begin{array}{l}\text { Integrated } \\
\text { environmental health } \\
\text { impact assessment }\end{array}$ & $\begin{array}{l}\text { Imperial College } \\
\text { London }\end{array}$ & 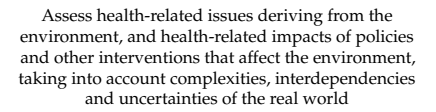 & $\begin{array}{l}\text { Different } \\
\text { scales } \\
\text { (local to } \\
\text { global) }\end{array}$ & UNSP & Yes \\
\hline Buonocore et al. [15] & 2016 & UNSP & $\begin{array}{l}\text { Air pollution (PM2.5, } \\
\text { NOx and SO2) }\end{array}$ & Premature deaths & Mitigation & $\begin{array}{l}\text { Nested } \\
\text { models }\end{array}$ & UNSP & Harvard University & $\begin{array}{l}\text { Assess reductions in NOx, PM2,5 and } \mathrm{CO}_{2} \text { and health } \\
\text { gains associated (Premature deaths avoided per year) } \\
\text { with offshore wind electricity }\end{array}$ & States & $\begin{array}{l}\text { New Jersey } \\
\text { and Maryland } \\
\text { (USA) }\end{array}$ & Yes \\
\hline Cai et al. [16] & 2018 & UNSP & Air pollution (PM2.5) & Premature deaths & Mitigation & $\begin{array}{l}\text { Nested } \\
\text { models }\end{array}$ & UNSP & $\begin{array}{l}\text { Joint Center for } \\
\text { Global Change } \\
\text { Studies }\end{array}$ & $\begin{array}{l}\text { Assess reductions in NOx, } \mathrm{PM} 2,5 \text { and } \mathrm{CO} 2 \text { and } \\
\text { associated health gains of carbon dioxide mitigation } \\
\text { in the electric powerg generation sector }\end{array}$ & $\begin{array}{c}\text { Country } \\
\text { (subre- } \\
\text { gions) }\end{array}$ & China & Yes \\
\hline Chen et al. [17] & 2014 & Heatwaves & Air temperature & Heat-related mortality & Mitigation & $\begin{array}{c}\text { Nested } \\
\text { models }\end{array}$ & UNSP & CSIRO & $\begin{array}{l}\text { Assess the impact of urban vegetation in the } \\
\text { reduction of heat related mortality rate }\end{array}$ & City & $\begin{array}{l}\text { Melbourne } \\
\text { (Australia) }\end{array}$ & Yes \\
\hline Chiabai et al. [18] & 2018 & $\begin{array}{c}\text { Heatwaves, } \\
\text { floods and } \\
\text { heavy rainfalls }\end{array}$ & $\begin{array}{l}\text { Urban heat islands, } \\
\text { flods and air } \\
\text { pollution (3 classes) }\end{array}$ & Multiples (non-specific) & UNSP & $\begin{array}{l}\text { Conceptual } \\
\text { framework }\end{array}$ & $\begin{array}{l}\text { "Ecosystems } \\
\text { enriched" river, } \\
\text { Pressure, State, } \\
\text { Exposure, Effect, } \\
\text { Action (eDPSEEA) }\end{array}$ & $\begin{array}{l}\text { BC3-Basque Centre } \\
\text { for Climate Change, } \\
\text { Spain }\end{array}$ & $\begin{array}{l}\text { Linking CC impacts and adaptation actions on } \\
\text { environment and assess how these actions could affect } \\
\text { human health through various ways of exposure }\end{array}$ & UNSP & UNSP & UNSP \\
\hline Diallo et al. [20] & 2017 & UNSP & $\begin{array}{l}\text { Air pollution (PM10, } \\
\text { NOx), noise }\end{array}$ & $\begin{array}{l}\text { DALY of sleep disorders and } \\
\text { annoyance }\end{array}$ & Mitigation & $\begin{array}{l}\text { Impact } \\
\text { assessment } \\
\text { tools }\end{array}$ & $\begin{array}{l}\text { Health impact } \\
\text { assessment (HIA), } \\
\text { Other environmental } \\
\text { assessment tools }\end{array}$ & $\begin{array}{l}\text { WHO (HAA), Conseil } \\
\text { fédéral Suisse (SA) }\end{array}$ & $\begin{array}{l}\text { Assess the impacts of different GHG } \\
\text { reduction measures }\end{array}$ & City & $\begin{array}{c}\text { Geneva } \\
\text { (Switzerland) }\end{array}$ & Yes \\
\hline Garcia Menendez et al. [21] & 2015 & UNSP & $\begin{array}{l}\text { Air pollution (Ozone, } \\
\text { PM2.5) }\end{array}$ & Mortality & $\begin{array}{l}\text { Mitigation } \\
\text { (GHG } \\
\text { reduction } \\
\text { scenarios) }\end{array}$ & $\begin{array}{l}\text { Nested } \\
\text { models }\end{array}$ & UNSP & $\begin{array}{l}\text { Massachusetts } \\
\text { Instituteo ef } \\
\text { Technology }\end{array}$ & $\begin{array}{l}\text { Allow an integrated analysis of the effects of } \mathrm{CC} \\
\text { mitigation measures on air pollution and health } \\
\text { co-benefits }\end{array}$ & Country & United States & Yes \\
\hline Haluza et al. [22] & 2012 & UNSP & $\begin{array}{l}\text { Air pollution (PM10, } \\
\text { NOx) }\end{array}$ & $\begin{array}{l}\text { Cardiovascular and respiratory } \\
\text { mortality }\end{array}$ & $\begin{array}{c}\text { Mitigation } \\
\text { (scenarios) }\end{array}$ & $\begin{array}{l}\text { Other method- } \\
\text { ological } \\
\text { approach }\end{array}$ & UNSP & $\begin{array}{c}\text { Institute of } \\
\text { Environmental } \\
\text { Health, Center for } \\
\text { Public Health, } \\
\text { Medical University } \\
\text { of Vienna }\end{array}$ & & Region & Upper Austria & Yes \\
\hline Houghton et al. [23] & 2012 & $\begin{array}{l}\text { Heatwaves, } \\
\text { floods }\end{array}$ & Air temperature & $\begin{array}{l}\text { Mortality (Cardiovascular, diabetes } \\
\text { and hypertension) }\end{array}$ & $\begin{array}{l}\text { Adaptation } \\
\text { and Mitigation }\end{array}$ & $\begin{array}{l}\text { Adaptation } \\
\text { tools }\end{array}$ & $\begin{array}{c}\text { Geospatial } \\
\text { Emerency } \\
\text { Managenent } \\
\text { Support System } \\
\text { (GEMSS) }\end{array}$ & $\begin{array}{l}\text { Texas Water } \\
\text { Development Board }\end{array}$ & & City & Austin (USA) & No \\
\hline Houghton [24] & 2011 & $\begin{array}{l}\text { Tornadoes, } \\
\text { hurricanes, } \\
\text { heat/drought, } \\
\text { and lightning }\end{array}$ & $\begin{array}{l}\text { Air temperature, } \\
\text { wind }\end{array}$ & Mortality, injuries & $\begin{array}{c}\text { Adaptation } \\
\text { and Mitigation }\end{array}$ & $\begin{array}{l}\text { Impact } \\
\text { Assessment } \\
\text { Tool }\end{array}$ & $\begin{array}{l}\text { Health Impact } \\
\text { Assessment }\end{array}$ & wно & $\begin{array}{l}\text { Assess climate change resilience in specific } \\
\text { building projects }\end{array}$ & City & $\begin{array}{l}\text { Houston } \\
\text { (USA) }\end{array}$ & No \\
\hline
\end{tabular}


Table 2. Cont.

\begin{tabular}{|c|c|c|c|c|c|c|c|c|c|c|c|c|}
\hline & & & & & & (a) & & & & & & \\
\hline Author & Year & $\begin{array}{c}\text { Climatic } \\
\text { Event }\end{array}$ & Exposure & Health Issue & $\begin{array}{l}\text { Strategy } \\
\text { (Adapta- } \\
\text { tion/Mitigation) }\end{array}$ & Tool Type & Name & Provider & Tool's Objective & $\begin{array}{l}\text { Scale of } \\
\text { Applica- } \\
\text { tion }\end{array}$ & Area & $\begin{array}{l}\text { Measure of } \\
\text { the Health } \\
\text { Effect }\end{array}$ \\
\hline Kwan et al. [25] & 2016 & UNSP & $\begin{array}{l}\text { Air pollution } \\
\text { (PM2.5), physical } \\
\text { activity, and road } \\
\text { crashes }\end{array}$ & Mortality & Mitigation & $\begin{array}{c}\text { Impact } \\
\text { Assessment } \\
\text { Tool }\end{array}$ & $\begin{array}{c}\text { Comparative Risk } \\
\text { Assessment }\end{array}$ & шно & $\begin{array}{l}\text { Assess the co-benefits of a mass rapid transit project } \\
\text { in terms of mortality reduction }\end{array}$ & City & $\begin{array}{c}\text { Kuala Lumpur } \\
\text { (Malasisia) }\end{array}$ & Yes \\
\hline Li and Crawford-Brown [26] & 2011 & UNSP & $\begin{array}{l}\text { Air pollution (PM2.5 } \\
\text { and PM10) }\end{array}$ & $\begin{array}{l}\text { Cardiovascular and respiratory } \\
\text { (asthma, bronchitis) mortality }\end{array}$ & Mitigation & $\begin{array}{c}\text { Adaptation } \\
\text { tools }\end{array}$ & UNSP & US EPA & $\begin{array}{c}\text { Support decision-making using cost-benefit } \\
\text { comparisons and health co-benenit assessments of air } \\
\text { pollution reduction }\end{array}$ & City & $\begin{array}{c}\text { Bangkok } \\
\text { (Thailand) }\end{array}$ & Yes \\
\hline Lindsay et al. [27] & 2011 & UNSP & $\begin{array}{l}\text { Air pollution }\left(\mathrm{PM}_{10},\right. \\
\text { NO2, Co), hhysical } \\
\text { activity, road crash }\end{array}$ & $\begin{array}{l}\text { Cardiovascular and respiratory } \\
\text { (bronchitis) mortality }\end{array}$ & Mitigation & $\begin{array}{c}\text { Other method- } \\
\text { ological } \\
\text { approach }\end{array}$ & $\begin{array}{l}\text { Combination of tools } \\
\text { and survey data }\end{array}$ & $\begin{array}{l}\text { University of } \\
\text { Auckland }\end{array}$ & $\begin{array}{l}\text { Estimate the effects on health, costs, air pollution, } \\
\text { GHG emissions if shorrt trips were undertaken by } \\
\text { bicycle rather than car }\end{array}$ & Country & New Zealand & Yes \\
\hline Markandya et al. [28] & 2009 & UNSP & $\begin{array}{l}\text { Air pollution (CO2, } \\
\text { PM2.5) }\end{array}$ & $\begin{array}{l}\text { Mortality (cardiorespiratory disease } \\
\text { and lung cancer), acute } \\
\text { respiratatory infections }\end{array}$ & Mitigation & $\begin{array}{l}\text { Nested } \\
\text { models }\end{array}$ & $\begin{array}{l}\text { Three models } \\
\text { (POLES, GAINS, and } \\
\text { WHO Comparative } \\
\text { Risk Assessment) } \\
\end{array}$ & шно & $\begin{array}{l}\text { Assess modifications of particulate air pollution and } \\
\text { health effetetr resultitg from GHGG reduction measures } \\
\text { in the electricity generation sector }\end{array}$ & Countries & $\begin{array}{l}\text { European } \\
\text { Union, China } \\
\text { and India }\end{array}$ & Yes \\
\hline Perez et al. [29] & 2015 & UNSP & $\begin{array}{l}\text { Air pollution (PM2.5, } \\
\text { elemental carbon), } \\
\text { physical activity, } \\
\text { noise }\end{array}$ & Mortality (noise, air pollution); DALY & Adaptation & $\begin{array}{l}\text { Impact } \\
\text { assessment } \\
\text { tools }\end{array}$ & $\begin{array}{l}\text { Health Impact } \\
\text { Assessment }\end{array}$ & шно & $\begin{array}{l}\text { Assess the health impacts of local } \mathrm{CC} \text { mitigation } \\
\text { policies in the transport sector }\end{array}$ & City & $\begin{array}{c}\text { Bale } \\
\text { (Switzerland) }\end{array}$ & Yes \\
\hline Sarigiannis et al. [30] & 2017 & UNSP & $\begin{array}{l}\text { Air pollution (PM2.5, } \\
\text { PM10, NO2, and } \\
\text { benzene) }\end{array}$ & Mortality, DALY & $\begin{array}{l}\text { Mitigation } \\
\text { (GHG } \\
\text { reduction) }\end{array}$ & $\begin{array}{l}\text { Nested } \\
\text { models }\end{array}$ & UNSP & $\begin{array}{l}\text { Aristotle University } \\
\text { of Thessaloniki }\end{array}$ & $\begin{array}{l}\text { Assess health co-benefits associated with GHG } \\
\text { reduction policies in transportation }\end{array}$ & City & $\begin{array}{c}\text { Thessaloniki } \\
\text { (Greece) }\end{array}$ & Yes \\
\hline Smith and Haigler [31] & 2008 & $\begin{array}{c}\text { Rising } \\
\text { temperatures }\end{array}$ & $\begin{array}{l}\text { Air pollution } \\
\text { (methane, } \mathrm{CO} 2)\end{array}$ & DALY, years of life lost (YLL) & Mitigation & $\begin{array}{l}\text { Other method- } \\
\text { ological } \\
\text { approaches }\end{array}$ & UNSP & wно & $\begin{array}{l}\text { Assess health co-benefits associated with GHG } \\
\text { reduction policies in the energy sector }\end{array}$ & Country & China & Yes \\
\hline Thompson et al. [33] & 2016 & UNSP & $\begin{array}{l}\text { Air pollution (ozone } \\
\text { and PM2.5) }\end{array}$ & $\begin{array}{l}\text { Mortality risk, morbidity (hospital } \\
\text { admissions, memergency room visists, } \\
\text { lost school days, acute respiratory } \\
\text { symptoms, acute myocardial } \\
\text { infarction (nonfatal heart attacks) and } \\
\text { acute bronchitis) }\end{array}$ & Mitigation & $\begin{array}{l}\text { Nested } \\
\text { models }\end{array}$ & UNSP & US EPA (BenMAP) & $\begin{array}{l}\text { Assess health and monetary impacts of a carbon } \\
\text { policy at the subnational scale }\end{array}$ & Region & $\begin{array}{l}\text { Northeast } \\
\text { USA (17 } \\
\text { States) }\end{array}$ & Yes \\
\hline Tobollik et al. [34] & 2016 & UNSP & $\begin{array}{l}\text { Air pollution (PM2.5, } \\
\text { elemental carbon) } \\
\text { and noise }\end{array}$ & $\begin{array}{l}\text { YLL, years lived with disability } \\
\text { (YLD) }\end{array}$ & Mitigation & $\begin{array}{l}\text { Impact } \\
\text { assessment } \\
\text { tool }\end{array}$ & $\begin{array}{l}\text { Health Impact } \\
\text { Assessment }\end{array}$ & wно & $\begin{array}{l}\text { Assess the health co-benefits of local CC mitigation } \\
\text { policies in the transport sector }\end{array}$ & City & $\begin{array}{l}\text { Rotterdam } \\
\text { (Netherlands) }\end{array}$ & Yes \\
\hline Tuomisto et al. [35] & 2015 & UNSP & Air pollution (PM2.5) & Mortality, DALY & Mitigation & $\begin{array}{l}\text { Nested } \\
\text { models }\end{array}$ & Opasnet & $\begin{array}{l}\text { URGENCHE (EU } \\
\text { FP7 project) }\end{array}$ & $\begin{array}{l}\text { Estimate health impacts of emissions due to heat and } \\
\text { power consumption of buildings sand give guidance } \\
\text { on different climate mititigation options }\end{array}$ & Cities & $\begin{array}{l}\text { Bâle } \\
\text { (Switzerland), } \\
\text { Kuopio } \\
\text { (Finland) }\end{array}$ & Yes \\
\hline Williams et al. [36] & 2018 & UNSP & $\begin{array}{l}\text { Air pollution (PM2.5, } \\
\text { NO2 and ozone) }\end{array}$ & YLL & Mitigation & $\begin{array}{l}\text { Nested } \\
\text { models }\end{array}$ & UNSP & King's College & $\begin{array}{l}\text { Assess health co-benefits of different CC mitigation } \\
\text { actions in the energy sector }\end{array}$ & Country & Great Britain & Yes \\
\hline Wolkinger et al. [37] & 2018 & UNSP & $\begin{array}{l}\text { Air pollution (PM2.5, } \\
\text { PM10 and NO2) }\end{array}$ & $\begin{array}{l}\text { Mortality, hospital admissions, and } \\
\text { years lived with disability for } \\
\text { cardiovasular and respiratory } \\
\text { diseases. Physical activity. }\end{array}$ & Mitigation & $\begin{array}{l}\text { Nested } \\
\text { models }\end{array}$ & UNSP & $\begin{array}{l}\text { Center for Climate } \\
\text { and Global Change, } \\
\text { (Austria) }\end{array}$ & $\begin{array}{l}\text { Allow a detailed health and macroeconomic } \\
\text { assessment of CC adaptation policies }\end{array}$ & Cities & $\begin{array}{l}\text { Graz, Vienna } \\
\text { and Linz } \\
\text { (Austria) }\end{array}$ & Yes \\
\hline Woodcock et al. [38] & 2009 & UNSP & $\begin{array}{l}\text { Air pollution (PM2.5, } \\
\text { PM10) }\end{array}$ & YLL, YLD, DALY, and mortality & Mitigation & $\begin{array}{l}\text { Impact } \\
\text { assessment } \\
\text { tool }\end{array}$ & $\begin{array}{c}\text { Comparative Risk } \\
\text { Assessment }\end{array}$ & шно & $\begin{array}{l}\text { Compare the health effects of different mitigation } \\
\text { scenarios with a reference situation }\end{array}$ & Cities & $\begin{array}{c}\text { New Delhi } \\
\text { (India); } \\
\text { London, } \\
\text { (United } \\
\text { Kingdom) }\end{array}$ & Yes \\
\hline Zhang et al. [39] & 2016 & UNSP & Air pollution (PM2.5) & Mortality and morbidity & Mitigation & $\begin{array}{l}\text { Nested } \\
\text { models }\end{array}$ & UNSP & $\begin{array}{l}\text { Copernicus Institute } \\
\text { of Sustainable } \\
\text { Development } \\
\text { (Utrecht University) }\end{array}$ & $\begin{array}{l}\text { Assess the potential for energy savings and emission } \\
\text { mitigation of air rollution from China's cementh } \\
\text { industry and quantify he health co-benefit linked } \\
\text { with air pollution reduction in this sector }\end{array}$ & Regions & $\begin{array}{l}\text { China (all } \\
\text { provinces) }\end{array}$ & Yes \\
\hline
\end{tabular}


Table 2. Cont.

\begin{tabular}{|c|c|c|c|c|c|c|c|c|c|c|c|c|}
\hline \multicolumn{13}{|c|}{ (b) } \\
\hline Author & Year & $\begin{array}{c}\text { Climatic } \\
\text { Event }\end{array}$ & Exposure & Health Issue & $\begin{array}{l}\text { Strategy } \\
\text { (Adaptation, } \\
\text { Mitigation) }\end{array}$ & Tool Type & Name & Provider & Tool's Objective & Scale & Area & $\begin{array}{l}\text { Measure of } \\
\text { the Health } \\
\text { Effect }\end{array}$ \\
\hline Rudolph et al. [40] & 2018 & UNSP & UNSP & UNSP & Mitigation & $\begin{array}{l}\text { Conceptual } \\
\text { framework }\end{array}$ & $\begin{array}{l}\text { Climate, health, and } \\
\text { equity vulnerability } \\
\text { assessment }\end{array}$ & $\begin{array}{l}\text { Public Health } \\
\text { Institute Ceanter for } \\
\text { Climate Change and } \\
\text { Health }\end{array}$ & Assess health and climate vulnerabilities & UNSP & UNSP & UNSP \\
\hline UNFCCC [41] & 2011 & UNSP & UNSP & UNSP & UNSP & $\begin{array}{c}\text { Impact } \\
\text { assessment } \\
\text { tool }\end{array}$ & $\begin{array}{c}\text { Health Impact } \\
\text { Assessment }\end{array}$ & $\begin{array}{c}\text { WHO, Curtin } \\
\text { University WHO } \\
\text { Collaborating Centre }\end{array}$ & $\begin{array}{l}\text { Assess potential CC impacts and develop adaptation } \\
\text { responses to support governmental decision making }\end{array}$ & UNSP & UNSP & Yes \\
\hline WHO (Europe regional office) [42] & 2013 & UNSP & UNSP & UNSP & Adaptation & $\begin{array}{c}\text { Adaptation } \\
\text { tools }\end{array}$ & $\begin{array}{l}\text { Health and } \\
\text { adaptation costs }\end{array}$ & wно & $\begin{array}{l}\text { Support health adaptation planning in European } \\
\text { states by estimatitig health hand dadaptation costs and } \\
\text { efficiency of adaptation measures }\end{array}$ & Country & Europe & Yes \\
\hline WHO [44] & 2013 & UNSP & UNSP & UNSP & Adaptation & $\begin{array}{c}\text { Adaptation } \\
\text { tools }\end{array}$ & $\begin{array}{l}\text { Vulnerability and } \\
\text { adaptation } \\
\text { assessment }\end{array}$ & wHO & $\begin{array}{l}\text { Provide guidelines to improve the elaboration of } \\
\text { vulnerability and adapatation assessment and plan the } \\
\text { adaptation of the health sector (similat to Health } \\
\text { National adaptaptation processs) }\end{array}$ & Country & UNSP & Yes \\
\hline WHO [45] & 2014 & UNSP & UNSP & UNSP & Adaptation & $\begin{array}{c}\text { Adaptation } \\
\text { tools }\end{array}$ & $\begin{array}{c}\text { Health National } \\
\text { adaptation process }\end{array}$ & wно & $\begin{array}{l}\text { Ensure that the process of iteratively managing the } \\
\text { health risks of chimatete change is integrated into the } \\
\text { overall National Adaptation Plan process to achieve } \\
\text { the goals of healthy people in healthy communities }\end{array}$ & Country & $\begin{array}{c}\text { Directed to } \\
\text { developing } \\
\text { countries and } \\
\text { least- } \\
\text { developed } \\
\text { countries }\end{array}$ & $\begin{array}{c}\text { Yes } \\
\text { (indicators) }\end{array}$ \\
\hline Ontario government [46] & 2016 & UNSP & UNSP & UNSP & Adaptation & $\begin{array}{c}\text { Adaptation } \\
\text { tools }\end{array}$ & $\begin{array}{l}\text { Vulnerability and } \\
\text { adaptation } \\
\text { assessment }\end{array}$ & wно & $\begin{array}{l}\text { Support a resilient and adaptive public health system } \\
\text { to anticipaptet take into account, and attenuate the } \\
\text { emerging risks and impacts of CC (simliar to National } \\
\text { health adaptation process) }\end{array}$ & Province & $\begin{array}{l}\text { Ontario, } \\
\text { Canada }\end{array}$ & Yes \\
\hline
\end{tabular}

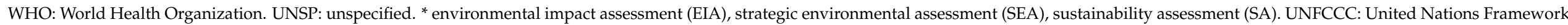

Convention on Climate Change. 
Environmental Assessments

The aim of environmental assessments is to identify and evaluate the potential consequences on the environment of proposed initiatives. In this context, environmental impact assessment (EIA) is used to assess the environmental impacts of projects while strategic environmental assessment (SEA) is applied to policies, plans, or programs. EIA and SEA processes are quite similar to that of HIA. EIA can be used to determine adaptation and mitigation measures that are favorable to both the environment and health. This implies that EIA is conducted in accordance with the European Union Directive (2014/52/EU), which broadened the scope of assessment by explicitly including human health among the topics to be addressed in an EIA. Similarly, SEA can be applied to select climate change adaptation or mitigation options to favor environmental protection and health promotion. To achieve this, conducting SEA needs to comply with the Directive 2001/42/EC of the European Parliament and of the Council and the SEA protocol to the Espoo Convention, both requiring human health issues to be taken into account when conducting a SEA.

\subsubsection{Adaptation tools}

Vulnerability and Adaptation (V\&A) Assessment

The WHO Executive Board has developed the Vulnerability and adaptation (V\&A) assessment. This is an approach to understand the current and future health risks related to climate change and thus develop strategies, policies, and measure to better address these risks [46]. It is a way to help involve the health sector in climate change adaptation efforts [50].

The objective of a V\&A assessment is to assist decision-makers in understanding the potential health risks attributable to climate change, in managing these risks, and in prioritizing policies and programmes to improve population health in a changing climate. A V\&A assessment involves the following five basic steps: (1) Define the frame and scope of the assessment; (2) Conduct the vulnerability and adaptation assessment; (3) Understand future impacts on health; (4) Prioritize and implement health protection regarding adaptation to climate change; (5) Establish an iterative process for managing and monitoring the health risks of climate change [44].

An example of an adaption tool is the Geospatial Emergency Management Support System (GEMSS), a browser-based tool. GEMSS is a platform developed by the Texas Water Development Board for integrating data linked to environmental, public health, and policy indicators related to climate change that can be visualized on the web. It makes it possible to combine the information in the form of several maps: climatic events (hot days, heavy rains), mortality, an indicator of vulnerability, climate-related policies. This tool has been used by the City of Austin to assess climate-health vulnerability [23], including adaptation and mitigation policies.

\section{Health National Adaptation Process (HNAP)}

Developed by the WHO, and based on the United Nations Framework Convention on Climate Change (UNFCCC) climate change agenda, the HNAP's objective is to ensure that the iterative management process of health risks resulting from climate change is integrated into the overall national adaptation plan (NAP) process to achieve the goal of a healthy population in healthy communities. This process consists of the following four elements (A-D), which are subdivided into eleven steps:

\section{(A) Lay the groundwork and address gaps in undertaking the HNAP process:}

Step 1. Aligning the health adaptation planning process with the national process for developing a NAP;

Step 2. Taking stock of available information;

Step 3. Identifying approaches to address capacity gaps and weaknesses in HNAP implementation.

(B) HNAP preparatory elements: 
Step 4. Conducting a V\&A assessment in the health sector, including short- and long-term needs in the context of development priorities;

Step 5. Examining the implications of climate change for development goals, legislation, strategies, policies, and plans related to health;

Step 6. Developing a national health adaptation strategy that identifies priority adaptation options.

\section{(C) Implementation strategies:}

Step 7. Elaborating an implementation strategy for operationalizing HNAPs and incorporating climate change adaptation in health-related planning processes at all levels, including strengthening the capacity for conducting future HNAPs;

Step 8. Promoting coordination and synergies with the NAP process, especially with sectors affecting health, and with multilateral environmental agreements.

\section{(D) Reporting, monitoring, and review:}

Step 9. Following-up and reviewing the HNAP to assess progress, effectiveness, and gaps; Step 10. Updating the health component of the NAPs in an iterative manner;

Step 11. Communicating and reporting on the progress and effectiveness of the HNAP implementation [45].

Economic Assessment Tool-Health and Adaptation Costs

The WHO Regional Office for Europe has developed this economic analysis tool to facilitate the planning of adaptation measures to protect health from the adverse effects of climate change in the Member States of the European Region. The tool allows users to estimate health and adaptation costs through three dimensions: (1) the costs related to damage to health caused by climate change; (2) the costs for adaptation in various sectors to protect health from such damage and (3) the efficiency of adaptation measures including avoided health costs [42]. The process for assessing health damage and adaptation costs involves four steps. Step (i) Define the scope of the assessment, involves deciding on the type of analysis and specifying the following elements: the level of application of the tool, the types of disease to be incorporated, the population groups concerned and the period of the analysis. Step (ii) Methods, data, sources, and analysis, consists of estimating both health damage costs and adaptation costs. It involves understanding methods, identifying data and sources, collecting data and inserting them in Excel sheets, conducting a sensitivity analysis, and analyzing results. Step (iii) Compare damage and adaptation costs, involves conducting a crude cost-benefit assessment. Step (iv) Present results, considers the target audience and communication needs.

\subsubsection{Nested Models}

Nested models have been defined and used to assess the effect on health of greenhouse gas (GHG) reduction policies in different sectors (transport, energy, industry, or building), as well as in urban planning (greening policies, in [17]). The energy sector is the most studied, and nested models have been applied regionally to study the energy sector in different jurisdictions such as in China [16,28], Europe [28,36], and the United States [15]. The other applications are linked with building emissions [12,35], with public transport in municipalities [30], or in the cement industry [39]. Finally, some studies assess the global effects of national or subnational carbon policies [21,33,37]. These type of tools have the same general structure and consist of a suite of several nested modeling modules: different mitigation scenarios, a model converting these scenarios into modifications in GHG emissions (NOx, CO2, PM, or ozone), a model estimating resulting atmospheric pollutant concentrations, and finally a health effects assessment model (i.e., BenMAP, [12]). These ensembles of models also include some tools already developed, such as the Health Economic Assessment Tool, developed by the WHO to estimate economic and health benefits of different policies, or HIA and comparative risk assessment [28]. Nested models are tools that allow for the prediction of the health impacts of different adaptation or 
mitigation strategies, although these strategies are mainly hypothetical and based on global policy objectives (such as the Paris Agreement, for example).

\subsubsection{Conceptual Frameworks}

This review of the scientific and grey literature revealed three examples of conceptual frameworks of interest as tools for integrating health into climate change adaptation/mitigation policies.

The first one is the "Ecosystems enriched" Driver, Pressure, State, Exposure, Effect, Action (eDPSEEA). Chiabai et al. [18] used the eDPSEEA framework to link climate change, adaptation actions and health by focusing on the potential health effects of changes in green spaces. In the proposed eDPSEEA conceptual framework, the driver is climate change caused by GHG emissions and concentrations. The pressures are represented by temperature and precipitation patterns, heat, air pollution and extreme weather events. This contributes to a potential change in the state of the environment, producing alterations in the functioning of the ecosystem that will then affect the terrestrial distribution of natural areas, and the regulation of the ecosystem services they provide in the short and long term. The state is characterized by 6 types of ecosystemic services that can affect the use or perception of a site through exposure (exposure): urban heat islands effect, air pollution, water cycle regulation, social environment, recreation, tourism, and microbiome. Based on a set of contextual factors (socioeconomic status, health status, culture, attitudes, beliefs, and environmental factors), these changes may have a direct or indirect, positive or negative impact on health (effect). Any intervention affecting green spaces and population exposure are considered an action.

The second one is a framework based on risk modelling to evaluate and provide scientific assessments of potential climate change adaptation measures. This framework includes three components: (1) knowledge synthesis; (2) Data storage and access; and (3) Quantitative risk modeling. Smith et al. [32] applied the framework to assess and compare the impacts of different adaptation scenarios related to water and food safety.

The third one is Climate, health and equity vulnerability assessment (CHEVA). CHEVA is a framework for assessing climate and health vulnerability. It includes four components for which a list of indicators is provided: (1) current and future physical threats of climate change; (2) population vulnerabilities including social determinants of health; (3) "adaptive capacity" that reflects individual and community-based resources that could counteract the negative impacts of climate change; and (4) health impact projections. A list of indicators has been developed to support the use of CHEVA. These indicators cover climate threats, population vulnerability, adaptive capacity, and resiliency [40].

\subsubsection{Other Methodological Approaches}

This review of the scientific and grey literature revealed three examples of methodological approaches of interest as tools for integrating health into climate change adaptation/mitigation policies.

\section{Participatory approach}

The participatory approach is a way to mobilize and involve the community in the fight against climate change. This approach has been used to assess the effects on the quality of life and well-being of residents and users of several pilot projects on the reduction of urban heat islands in the Montréal area. The assessment was based on four criteria: beauty, comfort, coolness and security [13].

\section{Mixed Methods}

Mixed methods refer to methods that combine different analytical approaches (survey data, literature review, models, and tools). Haluza et al. [22] applied a mixed-methods approach in the energy sector to estimate the health impacts of a shift from light fuel to residential wood-burning in one state in Austria. The methodological approach combines 
modeling to estimate emissions of air pollutants with reviewing epidemiological studies to assess health impacts (literature review).

In the transport sector, Lindsay et al. [27] used a combination of survey data and tools to study the impact of shifting urban transport from cars to bicycles on health, air pollution, and GHG emissions, in New Zealand. The following data sources and tools were used: the New Zealand Household Travel Survey, the Vehicle Emissions Prediction Model, the Health and Pollution in New Zealand study, the WHO Health Economic Assessment Tool (HEAT), and the Cycling injury/death data and 'safety in numbers' [27].

Finally, Smith et al. [31] presented a set of scoping methods to quickly assess cobenefits for interventions in the energy sector.

\section{Discussion}

The tools that were found most frequently in this scoping review are the nested models (12 of 35); these were applied to assess the health impacts of mitigation options on different sectors such as transport, energy, industry, or building, as well as in urban planning. Health Impact Assessments were also frequently identified (6/35) and were applied to specific building projects, alternative transportation options or in urban planning.

Among the identified tools, several have a similar structure. For example, the family of impact assessments such as EIA, SEA, and HIA comprises similar tools to aid decisionmaking processes, but the tools differ in the scope of impacts analyzed. Environmental assessments (EIA and SEA) focus on the impacts of proposed policies or projects on the physical environment, while HIA analyzes the consequences of these policies and projects on physical, social, and economic determinants of health. EIA, SEA, and HIA are prospective tools based on methodologies that attempt to integrate the concerns of different stakeholders' groups into the assessment process [47]. This means that an interdisciplinary and intersectoral scientific approach could be needed to evaluate the public health benefits of climate change adaptation or mitigation policies. Indeed, HIA is a combination of methods and tools. One of the differences between HIA and CRA is the object of analysis, which is a policy, program or project in the case of an HIA and a substance or exposure in the case of a CRA. A CRA can be integrated into an HIA framework.

The tools used could be different depending on the strategy chosen to face climate change consequences (adaptation or mitigation). Some tools tend to be dedicated to adaptation contexts such as V\&A assessment, Health National Adaptation Process, or health and adaptation costs. In contrast, nested models are also almost exclusively $(11 / 12$ studies) used to assess mitigation scenarios in prospective studies.

The scale of application also differs among the different tools identified. Nested models are adaptable to varying scales of study, and this flexibility means that they are applied to both city, national-level, or supranational contexts, depending on the policy. Some tools such as vulnerability assessment, also allow an application at different scales. Some methods, such as HIA, are applied mostly at a local scale (city- or project-level). However, it should be noted that these tools are not designed specifically to be applied at a particular scale and might also be applied at other scales. It is worth noting that studies identified in the scientific literature are mainly conducted in wealthier nations and regions, in Europe, North America, and Asia. Few case studies have been conducted in developing countries. Some studies were conducted in India, Malaysia, and Thailand, but no studies were reported in Africa, the Middle East or in South America. However, these regions may suffer greater mortality risks due to climate change [51,52].

The robustness of data is also of primary importance to provide robust tools. Practitioners need to rely on robust data from health networks and emissions monitoring networks in place. Quantification of health impacts is often a difficult exercise due to the availability of valid data and rigorous analytical methods for predicting these impacts [53,54].

Some limitations in the application of these tools are linked with the accessibility of data. The use of impact assessment tools, adaptation tools, and nested models all depend upon access to a lot of different data relating to impact quantification, potential 
health impacts or scenario assessment, as well as data on climatic or health conditions or socioeconomic profiles of the population.

This review shows that in recent years there has been increased interest in the topic of developing tools to better manage health issues in adaptation and mitigation strategies. This subject emerged as a scientific topic at the end of the 2000s, with an increase in the number of publications in recent years. Nested models have also been increasingly used since 2014; these complete the other groups of tools that emerged in the 2000s.

In a context of a health crisis such as the Covid-19 pandemic, which has led to the lockdown of billions of people, to dramatic decreases in transport emissions and industrial emissions in the main GHG emitting countries [55-57], the methods identified in this review could provide an interesting basis for assessing the health co-benefits of such rapid mitigation measures. Some of the methods identified, such as nested models could allow for the assessment of the short-term health effects of a decrease in transport emissions in a region (such as in Lombardy, Catalunya, or in the greater Paris region) or across a greater geographical area such as a country (e.g., China, [58]) or an entire continent. From another perspective, some actions taken during the COVID-19 pandemic could be favorable to the climate. In this context, some tools identified in this work, such as environmental impact assessment and HIA, can help optimize the health benefits of these actions before their implementation with a view to combating climate change.

This literature review, as is often the case in bibliographic research, has some limitations. Despite a rigorous methodological approach, it is possible that some relevant articles were not identified. There are three reasons for this. The first is that the keywords used for the research may not have been included in the titles or abstracts of relevant documents. The second is the exclusion of publications in languages other than French and English. Finally, the third is that this literature review focused only on the documentation available online and thus excluded all other documents that were not published online. $\mathrm{T}$ One additional limitation is that the choice of keywords used in the literature search could have disproportionately favored some tools in the results, as for example, the term "health impact assessment" that was included in the keywords search. However, this keyword was selected in keeping with this review's primary aim: to identify the tools that assess the health impacts of climate change. Removing this term from the literature review would have also led to missing some articles directly related to the objectives of this review. One limitation is that no analysis of the effectiveness of the tools identified was made, as that was judged to be beyond the scope of this review. Despite these limitations, we believe that this work helps to highlight several existing tools and methods that enable the integration of health concerns into the development of climate change adaptation and mitigation policies and strategies.

As suggested, an analysis of the effectiveness of these tools should be conducted in further studies. Moreover, the transferability of the tools identified in different jurisdictions (federal, provincial, or municipal) should be made in order to better identify the facilitating factors and the barriers for their application (such as, for example, political commitment, working in silos, knowledge, and skills). Uncertainties in climate change projections are linked with climate feedback, regional changes, or the strength of equilibrium climate sensitivity [59]. A better estimation and reduction of these uncertainties (and those linked with the quantification of health impacts) is also needed to refine and better approximate the quantification of health impacts.

\section{Conclusions}

Different countries, regions and cities around the world are implementing policies and strategies to adapt to climate change and mitigate its effects. This scoping review allowed for the identification of a total of 35 studies ( 28 from the scientific literature and 7 from the grey literature) that present tools and methods that were designed to promote or integrate health into climate change adaptation and mitigation policies or strategies. An increasing interest in this topic in the scientific literature was also noted since the beginning 
of the 2010s. The methods and tools that were most frequently found in this review are the nested models (12 of 35) and HIA (6 of 35) that were used to assess the health impacts of mitigation and adaptation options on different sectors such as transport, energy, industry or building, as well as in urban planning. Further studies should include additional analyses of the effectiveness of the tools and methods identified, as well as a quantification of the uncertainties linked with climate change projections and health impact estimates.

Author Contributions: Conceptualization, T.A.D. and I.D.; methodology, I.D.; formal analysis, I.D.; writing—original draft preparation, T.A.D. and I.D.; writing—review and editing, T.A.D., O.B., M.K. and I.D.; supervision, T.A.D.; project administration, O.B. All authors have read and agreed to the published version of the manuscript.

Funding: This research received no external funding.

Institutional Review Board Statement: Not applicable.

Informed Consent Statement: Not applicable.

Data Availability Statement: Data sharing not applicable.

Acknowledgments: The authors wish to thank the library services of the Institut national de sante publique du Québec (INSPQ) and Frédéric Bergeron from the Université Laval's library for their help with the implementation of the literature research strategy.

Conflicts of Interest: The authors declare no conflict of interest.

\section{References}

1. Watts, N.; Adger, W.N.; Agnolucci, P.; Blackstock, J.; Byass, P.; Cai, W.; Chaytor, S.; Colbourn, T.; Collins, M.; Cooper, A.; et al. Health and climate change: Policy responses to protect public health. Lancet 2015, 386, 1861-1914. [CrossRef]

2. Awuor, L.; Meldrum, R.; Liberda, E.N. Institutional Engagement Practices as Barriers to Public Health Capacity in Climate Change Policy Discourse: Lessons from the Canadian Province of Ontario. Int. J. Environ. Res. Public Health 2020, $17,6338$. [CrossRef]

3. Hobbhahn, N.; Fears, R.; Haines, A.; Ter Meulen, V. Urgent action is needed to protect human health from the increasing effects of climate change. Lancet Planet. Health 2019, 3, e333-e335. [CrossRef]

4. Patz, J.A.; Frumkin, H.; Holloway, T.; Vimont, D.J.; Haines, A. Climate Change: Challenges and opportunities for global health. JAMA 2014, 312, 1565-1580. [CrossRef] [PubMed]

5. Chan, M. Achieving a cleaner, more sustainable, and healthier future. Lancet 2015, 386, e27-e28. [CrossRef]

6. Young, I.; Smith, B.A.; Fazil, A. A systematic review and meta-analysis of the effects of extreme weather events and other weather-related variables on Cryptosporidium and Giardia in fresh surface waters. J. Water Health 2015, 13, 1-17. [CrossRef]

7. Haines, A. Health co-benefits of climate action. Lancet Planet. Health 2017, 1, e4-e5. [CrossRef]

8. Hoegh-Guldberg, O.; Jacob, D.; Taylor, M.; Bolaños, T.G.; Bindi, M.; Brown, S.; Camilloni, I.A.; Diedhiou, A.; Djalante, R.; Ebi, K.; et al. The human imperative of stabilizing global climate change at $1.5^{\circ} \mathrm{C}$. Science 2019, 365, eaaw6974. [CrossRef] [PubMed]

9. Maibach, E.W.; Sarfaty, M.; Mitchell, M.; Gould, R. Limiting global warming to 1.5 to $2.0^{\circ} \mathrm{C}-\mathrm{A}$ unique and necessary role for health professionals. PLoS Med. 2019, 16, e1002804. [CrossRef] [PubMed]

10. Dannenberg, A.L.; Rogerson, B.; Rudolph, L. Optimizing the health benefits of climate change policies using health impact assessment. J. Public Health Policy 2020, 41, 139-154. [CrossRef] [PubMed]

11. Moher, D.; Liberati, A.; Tetzlaff, J.; Altman, D.G. PRISMA Group: Preferred reporting items for systematic reviews and metaanalyses: The PRISMA statement. BMJ 2009, 339, b2535. [CrossRef]

12. Abel, D.W.; Holloway, T.; Harkey, M.; Meier, P.; Ahl, D.; Limaye, V.S.; Patz, J.A. Air-quality-related health impacts from climate change and from adaptation of cooling demand for buildings in the eastern United States: An interdisciplinary modeling study. PLoS Med. 2018, 15, e1002599. [CrossRef]

13. Beaudoin, M.; Gosselin, P. An effective public health program to reduce urban heat islands in Québec, Canada. Rev. Panam. Salud Pública 2016, 40, 160-166. [PubMed]

14. Briggs, D.J. A framework for integrated environmental health impact assessment of systemic risks. Environ. Health Glob. Access Sci. Source 2008, 7, 1-17. [CrossRef]

15. Buonocore, J.J.; Luckow, P.; Fisher, J.; Kempton, W.; Levy, J. Health and climate benefits of offshore wind facilities in the Mid-Atlantic United States. Environ. Res. Lett. 2016, 11, 1-11. [CrossRef]

16. Cai, W.; Hui, J.; Wang, C.; Zheng, Y.; Zhang, X.; Zhang, Q.; Gong, P. The Lancet Countdown on PM 2.5 pollution-related health impacts of China's projected carbon dioxide mitigation in the electric power generation sector under the Paris Agreement: A modelling study. Lancet Planet. Health 2018, 2, e151-e161. [CrossRef] 
17. Chen, D.; Wang, X.; Thatcher, M.; Barnett, G.; Kachenko, A.; Prince, R. Urban vegetation for reducing heat related mortality. Environ. Pollut. 2014, 192, 275-284. [CrossRef]

18. Chiabai, A.; Quiroga, S.; Martinez-Juarez, P.; Higgins, S.; Taylor, T. The nexus between climate change, ecosystem services and human health: Towards a conceptual framework. Sci. Total Environ. 2018, 635, 1191-1204. [CrossRef]

19. Diallo, T.; Cantoreggi, N.; Simos, J. Co-bénéfices pour la santé des politiques urbaines relatives au changement climatique à l'échelon local: L'exemple de Genève. Environ. Risques Sante 2016, 15, 332-340. [CrossRef]

20. Diallo, T.; Cantoreggi, N.; Simos, J.; Christie, D.P.T.H. Is HIA the most effective tool to assess the impact on health of climate change mitigation policies at the local level? A case study in Geneva, Switzerland. Glob. Health Promot. 2017, 24, 5-15. [CrossRef]

21. Garcia-Menendez, F.; Saari, R.K.; Monier, E.; Selin, N.E. U.S. Air Quality and Health Benefits from Avoided Climate Change under Greenhouse Gas Mitigation. Environ. Sci. Technol. 2015, 49, 7580-7588. [CrossRef] [PubMed]

22. Haluza, D.; Kaiser, A.; Moshammer, H.; Flandorfer, C.; Kundi, M.; Neuberger, M. Estimated health impact of a shift from light fuel to residential wood-burning in Upper Austria. J. Expo. Sci. Environ. Epidemiol. 2012, 22, 339-343. [CrossRef] [PubMed]

23. Houghton, A.; Prudent, N.; Scott, J.E.; Wade, R.; Luber, G. Climate change-related vulnerabilities and local environmental public health tracking through GEMSS: A web-based visualization tool. Appl. Geogr. 2012, 33, 36-44. [CrossRef]

24. Houghton, A. Health Impact Assessments A Tool for Designing Climate Change Resilience Into Green Building and Planning Projects. J. Green Build. 2011, 6, 66-87. [CrossRef]

25. Kwan, S.C.; Tainio, M.; Woodcock, J.; Hashim, J.H. Health co-benefits in mortality avoidance from implementation of the mass rapid transit (MRT) system in Kuala Lumpur, Malaysia. Rev. Environ. Health 2016, 31, 179-183. [CrossRef]

26. Li, Y.; Crawford-Brown, D.J. Assessing the co-benefits of greenhouse gas reduction: Health benefits of particulate matter related inspection and maintenance programs in Bangkok, Thailand. Sci. Total Environ. 2011, 409, 1774-1785. [CrossRef] [PubMed]

27. Lindsay, G.; Macmillan, A.; Woodward, A. Moving urban trips from cars to bicycles: Impact on health and emissions. Aust. N. Z. J. Public Health 2011, 35, 54-60. [CrossRef] [PubMed]

28. Markandya, A.; Armstrong, B.G.; Hales, S.; Chiabai, A.; Criqui, P.; Mima, S.; Tonne, C.; Wilkinson, P. Public health benefits of strategies to reduce greenhouse-gas emissions: Low-carbon electricity generation. Lancet 2009, 374, 2006-2015. [CrossRef]

29. Perez, L.; Trüeb, S.; Cowie, H.; Keuken, M.P.; Mudu, P.; Ragettli, M.S.; Sarigiannis, D.A.; Tobollik, M.; Tuomisto, J.; Vienneau, D.; et al. Transport-related measures to mitigate climate change in Basel, Switzerland: A health-effectiveness comparison study. Environ. Int. 2015, 85, 111-119. [CrossRef] [PubMed]

30. Sarigiannis, D.A.; Kontoroupis, P.; Nikolaki, S.; Gotti, A.; Chapizanis, D.; Karakitsios, S. Benefits on public health from transportrelated greenhouse gas mitigation policies in Southeastern European cities. Sci. Total Environ. 2017, 579, 1427-1438. [CrossRef]

31. Smith, K.R.; Haigler, E. Co-Benefits of Climate Mitigation and Health Protection in Energy Systems: Scoping Methods. Annu. Rev. Public Health 2008, 29, 11-25. [CrossRef] [PubMed]

32. Smith, B.A.; Ruthman, T.; Sparling, E.; Auld, H.; Comer, N.; Young, I.; Lammerding, A.M.; Fazil, A. A risk modeling framework to evaluate the impacts of climate change and adaptation on food and water safety. Food Res. Int. 2015, 68, 78-85. [CrossRef]

33. Thompson, T.M.; Rausch, S.; Saari, R.K.; Selin, N.E. Air quality co-benefits of subnational carbon policies. J. Air Waste Manag. Assoc. 2016, 66, 988-1002. [CrossRef] [PubMed]

34. Tobollik, M.; Keuken, M.; Sabel, C.; Cowie, H.; Tuomisto, J.; Sarigiannis, D.; Künzli, N.; Perez, L.; Mudu, P. Health impact assessment of transport policies in Rotterdam: Decrease of total traffic and increase of electric car use. Environ. Res. 2016, 146, 350-358. [CrossRef] [PubMed]

35. Tuomisto, J.T.; Niittynen, M.; Pärjälä, E.; Asikainen, A.; Perez, L.; Trüeb, S.; Jantunen, M.; Künzli, N.; Sabel, C.E. Building-related health impacts in European and Chinese cities: A scalable assessment method. Environ. Health Glob. Access Sci. Source 2015, 14, 1-13. [CrossRef]

36. Williams, M.L.; Lott, M.C.; Kitwiroon, N.; Dajnak, D.; Walton, H.; Holland, M.; Pye, S.; Fecht, D.; Toledano, M.B.; Beevers, S.D. The Lancet Countdown on health benefits from the UK Climate Change Act: A modelling study for Great Britain. Lancet Planet. Health 2018, 2, e202-e213. [CrossRef]

37. Wolkinger, B.; Haas, W.; Bachner, G.; Weisz, U.; Steininger, K.; Hutter, H.P.; Delcour, J.; Griebler, R.; Mittelbach, B.; Maier, P.; et al. Evaluating Health Co-Benefits of Climate Change Mitigation in Urban Mobility. Int. J. Environ. Res. Public Health 2018, 15, 880. [CrossRef] [PubMed]

38. Woodcock, J.; Edwards, P.; Tonne, C.; Armstrong, B.G.; Ashiru, O.; Banister, D.; Franco, O.H.; Haines, A.; Hickman, R.; Lindsay, G.; et al. Public health benefits of strategies to reduce greenhouse-gas emissions: Urban land transport. Lancet 2010, 374, 1930-1943. [CrossRef]

39. Zhang, S.; Worrell, E.; Crijns-Graus, W.; Krol, M.; de Bruine, M.; Geng, G.; Wagner, F.; Cofala, J. Modeling energy efficiency to improve air quality and health effects of China's cement industry. Appl. Energy 2016, 184, 574-593. [CrossRef]

40. Rudolph, L.; Harrison, C.; Buckley, L.; North, S. Climate Change, Health, and Equity: A Guide for Local Health Departments; Public Health Institute: Oakland, CA, USA; American Public Health Association: Washington, DC, USA, 2018; p. 370.

41. United Nations Framework on Climate Change (UNFCCC). Human Health. In CGE Training Materials for Vulnerability and Adaptation Assessment; UNFCCC: Bonn, Germany, 2011; Chapter 8.

42. World Health Organization (WHO); Regional Office for Europe [ROE]. Climate Change and Health: A Tool to Estimate Health and Adaptation Costs; WHO-ROE: Copenhagen, Denmark, 2013. 
43. World Health Organization (WHO). Methods of Assessing Human Health Vulnerability and Public Health Adaptation to Climate Change; WHO: Geneva, Switzerland, 2003.

44. World Health Organization (WHO). Protecting Health from Climate Change: Vulnerability and Adaptation Assessment; WHO: Geneva, Switzerland, 2013.

45. World Health Organization (WHO). WHO Guidance to Protect Health from Climate Change through Health Adaptation Planning; WHO: Geneva, Switzerland, 2014.

46. Ebi, K.; Anderson, V.; Berry, P.; Paterson, J.; Yusa, A.; Gough, W.; Herod, K. Ontario Climate Change and Health Toolkit; Ministry of Health and Long-Term Care, Public Health Policy and Programs Branch: Toronto, ON, Canada, 2016; ISBN 978-1-4606-7703-2.

47. World Health Organization. Health in the Green Economy: Health Co-Benefits of Climate Change Mitigation-Transport Sector; WHO: Geneva, Switzerland, 2011.

48. Ezzati, M.; Van der Hoorn, S.; Rodgers, A.; Lopez, A.D.; Mathers, C.D.; Murray, C.J.; Comparative Risk Assessment Collaborating Group. Estimates of global and regional potentil health gains from reducing muliple major risk factors. Lancet 2003, 362, 271-280. [CrossRef]

49. Campbell-Lendrum, D.; Woodruff, R. Comparative Risk Assessment of the Burden of Disease from Climate Change. Environ. Health Perspect. 2006, 114, 1935-1941. [CrossRef]

50. Berry, P.; Enright, P.M.; Shumake-Guillemot, J.; Prats, E.V.; Campbell-Lendrum, D. Assessing Health Vulnerabilities and Adaptation to Climate Change: A Review of International Progress. Int. J. Environ. Res. Public Health 2018, 15, 2626. [CrossRef]

51. Carleton, T.; Delgado, M.; Greenstone, M.; Houser, T.; Hsiang, S.; Hultgren, A.; Jina, A.; Kopp, R.E.; McCusker, K.; Nath, I.; et al. Valuing the Global Mortality Consequences of Climate Change Accounting for Adaptation Costs and Benefits (No. w27599); National Bureau of Economic Research: Cambridge, MA, USA, 2020.

52. Li, L.; Jiang, C.; Murtugudde, R.; Liang, X.-Z.; Sapkota, A. Global Population Exposed to Extreme Events in the 150 Most Populated Cities of the World: Implications for Public Health. Int. J. Environ. Res. Public Health 2021, 18, 1293. [CrossRef]

53. Kemm, J. Chapter-Quantitative assessment. In Health Impact Assessment_Past Achievement, Current Understanding, and Future Progress; Kemm, J., Ed.; Oxford University Press: Oxford, UK, 2013; pp. 25-37.

54. Veerman, J.; Barendregt, J.; MacKenbach, J.P. Quantitative health impact assessment: Current practice and future directions. J. Epidemiol. Community Health 2005, 59, 361-370. [CrossRef]

55. Forster, P.M.; Forster, H.I.; Evans, M.J.; Gidden, M.J.; Jones, C.D.; Keller, C.A.; LambolliD, R.D.; Le Quéré, C.; Rogelj, J.; Rosen, D.; et al. Current and future global climate impacts resulting from COVID-19. Nat. Clim. Chang. 2020, 10, 913-919. [CrossRef]

56. Liu, Z.; Ciais, P.; Deng, Z.; Lei, R.; Davis, S.J.; Feng, S.; Zheng, B.; Cui, D.; Dou, X.; Zhu, B.; et al. Near-real-time monitoring of global CO2 emissions reveals the effects of the COVID-19 pandemic. Nat. Commun. 2020, 11, 1-12. [CrossRef] [PubMed]

57. Ching, J.; Kajino, M. Rethinking Air Quality and Climate Change after COVID-19. Int. J. Environ. Res. Public Health 2020, $17,5167$. [CrossRef]

58. Chen, K.; Wang, M.; Huang, C.; Kinney, P.L.; Anastas, P.T. Air pollution reduction and mortality benefit during the COVID-19 outbreak in China. Lancet Planet. Health 2020, 4, e210-e212. [CrossRef]

59. Brient, F. Reducing Uncertainties in Climate Projections with Emergent Constraints: Concepts, Examples and Prospects. Adv. Atmos. Sci. 2020, 37, 1-15. [CrossRef] 